\title{
TUNABLE PHOTONIC STRUCTURES BASED ON SILICON AND LIQUID CRYSTALS
}

\author{
Tatiana S. Perova ${ }^{*}$, Vladimir A. Tolmachev ${ }^{\mathrm{a}, \mathrm{b}}$, Ekaterina V. Astrova ${ }^{\mathrm{b}}$ \\ ${ }^{a}$ Department of Electronic and Electrical Engineering, University of Dublin, Trinity College, Dublin \\ 2, Ireland; \\ ${ }^{\mathrm{b}}$ Ioffe Physicotechnical Institute, Russian Academy of Sciences, St. Petersburg, 194021 Russia
}

\begin{abstract}
This paper is focused on the design, fabrication and characterization of the conventional and tunable photonic devices based on grooved silicon, serving as one-dimensional (1D) photonic crystal. The advantages of these photonic structures are as follows: the large refractive index contrast, in-plane moulding of the light flow, the possibility to fabricate a composite photonic structures by filling the grooves with a different compounds and compatibility with current semiconductor processing techniques. The optical properties of grooved Si structures were simulated using a transfer matrix method and gap map method and have been verified experimentally using FTIR microscopy. The air spaces in the basic silicon-air matrices were infiltrated with nematic liquid crystal E7. It is shown that the optical properties of the obtained composite 1D photonic crystals can be tuned by means of electro- and thermo-optical effects. Such a structures suit well for the various elements of the integrated optics and can serve as a building blocks for optical interconnects.
\end{abstract}

Keywords: 1D Photonic crystals, tunable photonic devices, FTIR microscopy, silicon microphotonics, photonic gap map

\section{INTRODUCTION}

Microphotonics has been recognised recently as one of the ways for the further development of communication and information technologies. Its elemental base is rapidly growing. It is recognised that the most important points for the development of new technologies are functionality, low cost and accessibility. From these points of view silicon is the most suitable material due to its low cost and well-developed CMOS processing ${ }^{1}$. Silicon-based optoelectronics could provide a low-cost solution for high-speed interconnects for data centres and other enterprises. The enabling technologies rely on fundamental building blocks such as silicon-based laser, amplifier and modulator ${ }^{1,2}$. Recently new areas of research in microphotonics were opened up due to elaboration of the theory and application of photonic crystals (PCs). Photonic crystals possess unique optical properties and can be used as reflectors, attenuators of the spontaneous atomic emission, devices for controlling emission in microcavities and others ${ }^{3-5}$. Furthermore, PCs have demonstrated their potential for application due to a number of micro-photonic devices utilized recently ${ }^{6}$. During the last decade particular attention has been paid to the development of devices based on one-dimensional (1D) PCs thanks to certain advantages of these devices over two- and three-dimensional PCs. These advantages are mainly related to their simpler fabrication and, therefore, low cost and in the possibility at the same time to have total reflection in a certain frequency (or wavelength) range for light at any incident angle, $\varphi$ and any polarization, i.e. to possess omni-directional bands (ODB) 7,8 .

For many possible applications in on-chip integrated devices (such as tunable filters, optical switches, waveguides and light emitters) the tuning of the photonic band gap (PBG) as well as the cavity spectral position during operation would be extremely important. As PBG structures consist of at least two alternating layers (components), tuning can be performed by changing the refractive index $(n)$ of one of the components. The approach suggested by Bush ${ }^{9}$ to use composite PCs with liquid crystal (LC) filler was the most attractive one for tunable photonic devices. The refractive index of an LC can vary depending on the state of the LC alignment. Since liquid crystals posses quite significant birefringence in the infrared range (up to $\Delta n=0.2$ ), variation of the LC filler refractive index has a much greater impact

*perovat@tcd.ie; phone 3531 896-1432; fax 3531 677-2442; http://www.mee.tcd.ie

Photonics: Design, Technology, and Packaging III

edited by Wieslaw Z. Krolikowski, Costas M. Soukoulis, Ping Koy Lam, Timothy J. Davis, Shanhui Fan, Yuri S. Kivshar Proc. of SPIE Vol. 6801, 68010W, (2008) · 0277-786X/08/\$18 doi: 10.1117/12.767324 
on the PBG shift than variation of silicon refractive index under heating or free carrier generation. Speaking about inplane silicon photonic elements, the band edge shift in 1D and 2D PCs has been demonstrated on grooved and macroporous silicon matrices infiltrated with nematic LC due to thermo- and electro-optical effects (phase transition in LC under heating ${ }^{10-13}$ and Freedericksz transition under applied voltage ${ }^{14-16}$ ). This paper is devoted to the design fabrication and characterisation of 1D PC-LC composite structures based on micro-structured grooved (110) Si for the moulding of in-plane light propagation. The theoretical approach based on photonic gap map presentation is elaborated for the analysis of the micro-cavity mode and omnidirectional reflection in 1D composite PCs. The results on calculations and experimental verification of the thermo-tuning and electro-tuning effects on the optical properties of 1D $\mathrm{PC}$ are summarised.

\section{ELABORATION OF THE GAP MAP METHOD FOR THE DESIGN AND ANALYSIS OF ONE- DIMENSIONAL PHOTONIC CRYSTALS}

The design of 1D PC is normally started with the selection of the wavelength, $\lambda$ of its operation. Then, the materials with corresponding refractive indices, $n 1$ and $n 2$ for suitable periodic structure fabrication will be chosen. Based on the above selection the thickness of components, $d 1$ and $d 2$ will be determined in accordance with Bragg conditions

$$
\lambda / 2=n 1 \cdot d 1+n 2 \cdot d 2
$$

where $n \cdot d$ is the optical thickness of the corresponding component.

When the optical thickness of each component equals $n \cdot d=\lambda / 4$ the maximum width of the stop-band (or PBG) is achieved for the required wavelength, $\lambda$. A few approaches can be used in order to determine the optical properties of 1D PC. One of these is to calculate the band diagrams ${ }^{17}$ for selected filling fraction $f=d 1 /(d 1+d 2)$, where $d 1+d 2=A$ is the lattice constant of PC. The second approach based on the gap map (GM) presentation of PBGs is described in Ref. ${ }^{5}$. For the gap map drawing in principal the band diagrams and/or the reflection spectra calculations can be used. The calculations of the reflection spectra, $R$ of PC with certain number of lattice periods, $m$ can be performed using Transfer Matrix method (TMM) ${ }^{18}$. It was shown previously that the GM approach is very useful for the design of the omnidirectional band (ODB) structures ${ }^{8,19}$ as well as tunable ${ }^{20}$ and composite ${ }^{21}$ photonic structures, since it allows quick and easy analysis of the structures on a large set of spectra (normally around 100) to be done practically on one plot.

In this paper we developed the gap map approach based on both aforementioned methods for the design and analysis of optical properties of 1D PCs. First, we used the band diagram (BD) calculations for ideal periodic structures with an infinite number of periods, $m$ and for one predicted filling factor. By doing this we can determine the position and width of the PBGs, including ODB. Then, the gap map (GM) method was applied which enabled us to select the proper filling fraction in the large range of possible numbers of $m$ and incidence angles. The advantage of the GM approach is that the design of ODB from the point of view of technical realisation, including the minimisation of the number of periods is possible. Finally, in the selected wavelength range of ODB and using the obtained optimal filling fraction the reflection spectra can be analysed. With this final procedure we can obtain information about the features of the reflection spectra, namely the maximum $R$ value, the band width, the sharpness of the stop-band edges, the presence of the interference fringes between stop-bands and others. The suggested approach is demonstrated on the two examples of 1D PC with medium and high refractive index contrast structures. In the first case the gap map of conventional photonic crystals with medium optical contrast is considered, while in the second case the gap map of high optical contrast PC with defect modes is analysed.

\subsection{The gap map of 1D PC with omnidirectional band}

Band diagram method

To create the band diagrams the Bloch solution was used in the same way as described by Yeh ${ }^{17}$. Consider a 1D PC with an infinite number of layers, this means that a translational symmetry along the periodic direction will lead to a Bloch solution of the field component. 


$$
\cos K \Lambda=\cos \left(k_{1 x} d 1\right) \cos \left(k_{2 x} d 2\right)-M \sin \left(k_{1 x} d 1\right) \sin \left(k_{2 x} d 2\right)
$$

where $\mathrm{K}$ is the Bloch wave vector, $k_{l x}$ and $k_{2 x}$ are the $x$ components of the wave vectors $k_{1}$ and $k_{2}$ of the first and second constituent layers respectively. The parameter $M$ is dependent on the polarization:

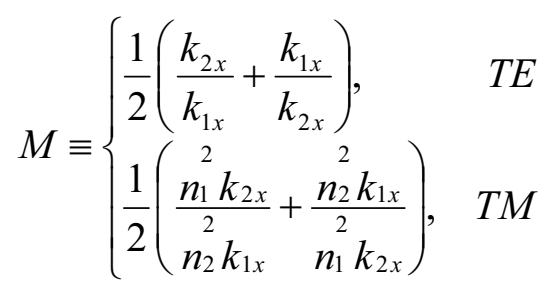

We assumed that the medium through which the incoming and outgoing light beams pass is air. The absence of light absorption in all of the layers is also assumed. The BDs of fundamental PBG for the photonic structures of medium optical contrast, $n 1 / n 2=1.5 / 3.42$ with optimum filling fraction of the first component at 0.695 are demonstrated in Fig. $1 \mathrm{a}$ as a function of the normalized frequency, i.e. A/ $\lambda$. In this case the overlapping of TM and TE polarization PBGs reveals an ODB. With a range of NF of $0.23-0.3$ this gives a $\triangle \mathrm{NF}$ of 0.07 . In general, ODB is defined by the range of overlapping of PBGs calculated for $0^{\circ}$ and maximal possible angle of incidence for TM and TE modes. Since the TE mode is wider than the TM one, the region of ODB is formed in fact from the width of TM mode in the range of $\varphi$ from $0^{\circ}$ to $85^{\circ}$ (Fig. 1 a).
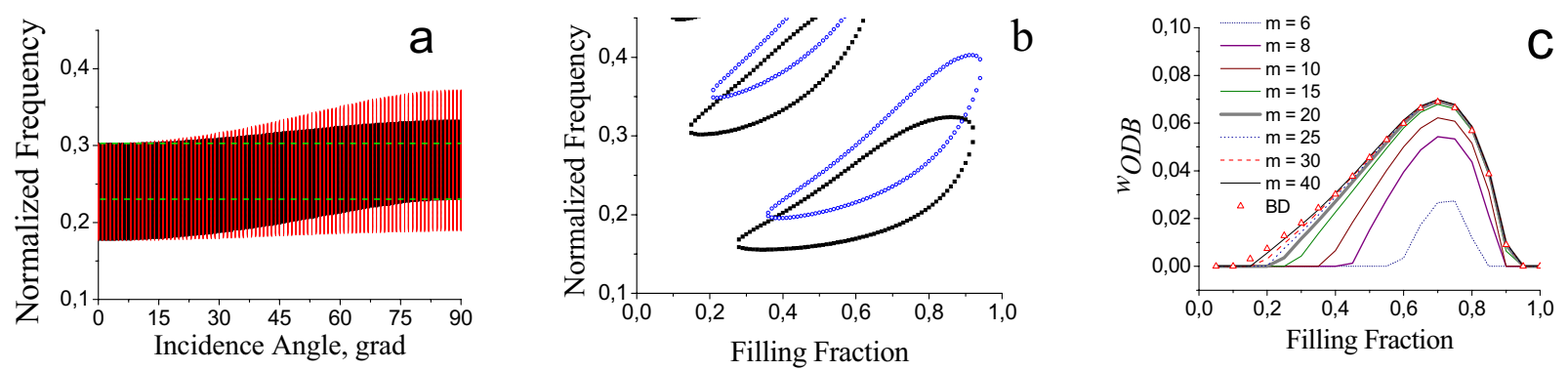

Fig.1. a) BD for TE and TM polarizations of a $1 \mathrm{D}$ PC with medium optical contrast $n 1 / n 2=1.5 / 3.42$ and filling fraction $f=$ 0.695 overlapped with the ODB indicated with two dash lines, b) gap map with the primary PBG region for $0^{\circ}$ (solid square) and $85^{\circ}$ (open circle) incident angles for TM polarization with $m=10$. c) Width of the omnidirectional band, $w_{O D B}$ versus the filling fraction over a number of periods using GM method and BD method for infinite number of periods.

\section{Gap Map calculation}

To create a GM first one must calculate the reflection spectrum of the PC structure. This is achieved by calculating the optical response of PCs using TMM. The 2 x 2 complex matrix S, describes the light propagation through the PC.

$$
S=\left[(I L)_{1}(I L)_{2}\right]^{m}
$$

where $I$ is the matrix that describes the interface between the two subscripted media, $L$ is the matrix that describes the subscripted media and $m$ is the number of periods. $I$ and $L$ are based upon the refractive index $n$ of the materials, the thickness $d$ of the materials, the incident angle, at which the light hits the surface and its polarization.

The reflection spectrum of the structure is calculated using Eqns. (4) and (5) for a range of filling fractions. 


$$
R=\left|\left[S_{1,0} / S_{0,0}\right]^{2}\right|
$$

For each of the reflection spectra, the region where the reflection was greater then 0.999 was recorded on the graph. Thus obtained calculated spectra were then plotted together with all the other filling fractions to give a graph that is called a gap map. The end result is displayed in Fig. $1 b$. Figure $1 b$ shows only two PBG regions (for $0^{\circ}$ and $85^{\circ}$ ) for TM mode with quite large regions of ODB for PC with $m=10$. The graph gives a number of PBGs where the reflection is greater then 0.999 as a general rule unless otherwise is stated for some specific cases.

To learn how the number of periods influences the formation of ODB region, the corresponding dependences of the ODB width, $w_{O D B}$ vs. filling fraction $f$ were plotted using data obtained from GM and BD calculations (see Fig. $1 c$ ). From these graphs one can see, that the parameters of the ideal PC (with an infinite number of periods) are practically the same for the PCs with 40 and greater number of periods. It can be also seen that a quite substantial ODB region is observed for the PC with six periods, which is important from the point of view of structure minimisation. This leads to the assumption that the analysis of GM, using the width for example $w_{O D B}=0.03-0.07$, allows us to determine the degree of ODB formation for PC, depending on the $m$ value and compared to infinite PC. Fig. $1 c$ shows that such a PC can be formed from a photonic structure with the number of periods $m>20$ and f varying in the range of 0.4 to 0.85 . Therefore, the selection of the construction of medium contrast PC with omni-band can be performed in a wide range using the range of $w_{O D B}$, number of periods and filling fraction.

The major limitation of the BD approach is that it assumes that there are an infinite number of periods. While in practice this is a good approximation of a PC with a very large number of periods namely 50, however creating these PCs with so many layers would be very challenging and not very practical. We must note that the gap map approach is very useful for practical applications since it can be applied to any number of periods over a wide range of values and is more suitable for the selection of the filling fraction because it allows quick and easy analysis of structures for the large set of spectra (normally around 100) to be done practically on one plot.

\subsection{The gap map of photonic structure with tunable defect mode}

The main feature of photonic crystals with defect mode is the relatively small number of lattice periods in the structure surrounding the defect. For example, for high- and low-contrast structures $m$ can be varied from 2 to 3 and from 10 to 15 , respectively. Therefore, using the TMM method is more preferable than the use of the dispersive equation.

Let us consider the design of the Fabri-Perrot micro-resonator (MR) with symmetrical Bragg reflectors (BRs) and with defect between them. The BRs with high optical contrast, $(n 1 / n 2=\mathrm{NH} / \mathrm{NL}=3.42 / 1)$, for example like micro-structured (grooved) silicon will be considered ${ }^{22}$. Due to this, the value of $m$ in these BRs should not be large, for example $m=3$. The wavelength of the resonator mode $\lambda_{\text {res }}$ (or wavenumber $v_{\text {res }}$ ) is the main parameter for the functioning the MR in the optical scheme. The tuning of the resonance peak position $\Delta \lambda_{\text {res }}=\lambda_{\text {res1 }}-\lambda_{\text {res2 }}$ can be achieved by means of the layer phase thickness $L_{\text {res }}$ variation:

$$
L_{r e s}=(2 \pi / \lambda) \cdot N \operatorname{def} \cdot \operatorname{Ddef} \cdot \cos \varphi
$$

It can be seen from Eqn. (6) that each of the three parameters, namely $N d e f$, Ddef and $\varphi$, may change $L_{r e s}$. At the same time we used the model with constant parameters of BRs. It is quite difficult to change the angle $\varphi$ as well as the geometrical size of defect quickly. Therefore, in most of the cases Ndef is changed in order to achieve the tuning of the resonance mode. Obviously, for a larger $\Delta N d e f=N 1$ def $-N 2$ def, a larger the shift of the resonant wavelength, $\sigma \lambda_{\text {res }}=\lambda_{\text {res } 1}$ $-\lambda_{\text {res2, }}$ can be obtained.

Then, we introduce the value $N d e f=1.5$ as a low refractive index defect, calculating the reflection spectra for $f$ values varied from 0.01 to 0.99 , and draw the gap map for 1D PC with the defect in the same manner as it was done for the gap map calculations. For the later procedure, only the wavenumbers which satisfied the criteria $R_{P B G}>0.999$ are selected, the rest of the wavenumbers are discarded. Then, for the tuning of the resonance mode in Eqn. (6) let us change the value of Ndef. This value is changed because it allows us to change the optical properties of the LC by means of the reorientation of its director. Based on new value of $N d e f=1.6$, we can draw the gap map with the new Ndef value (Fig. 2 
a) for the geometrical thicknesses $D d e f=1.25 \mathrm{DL}$ as well as for $D d e f$ equal to $1 \mathrm{DL}$ and $1.6 \mathrm{DL}$. The latter varies the defect size in Eqn. (6).

It can be seen from Fig. $2 a$, that when the defect size increases, the position of the resonance mode is red shifted. It is also evident that the width of the defect mode area (which is proportional to the width of the resonance peak of the resonator at its half height $\sigma \lambda_{\text {res }}$ ) depends on the value of $f$. Therefore, defect modes need to be compared when the width $\sigma \lambda_{\text {res }}$ is minimal for both values of the displacement parameter $N d e f$. Taking this into consideration, the $f$ values were then chosen. Figure $2 b$ shows spectra for $D d e f=1,1.25$ and $1.6 \mathrm{DL}$ with the corresponding $\mathrm{f}$ values $=0.2,0.23,0.3$. For example, with the application of a liquid crystal with $N d e f$ between 1.5 and 1.6 in the microresonator with $D d e f=1.6 \mathrm{DL}$, using the non-optimal $f$ value of 0.2 would result in a wider $\sigma \lambda_{\text {res }}$, i.e. a worse quality factor of the resonance peak.
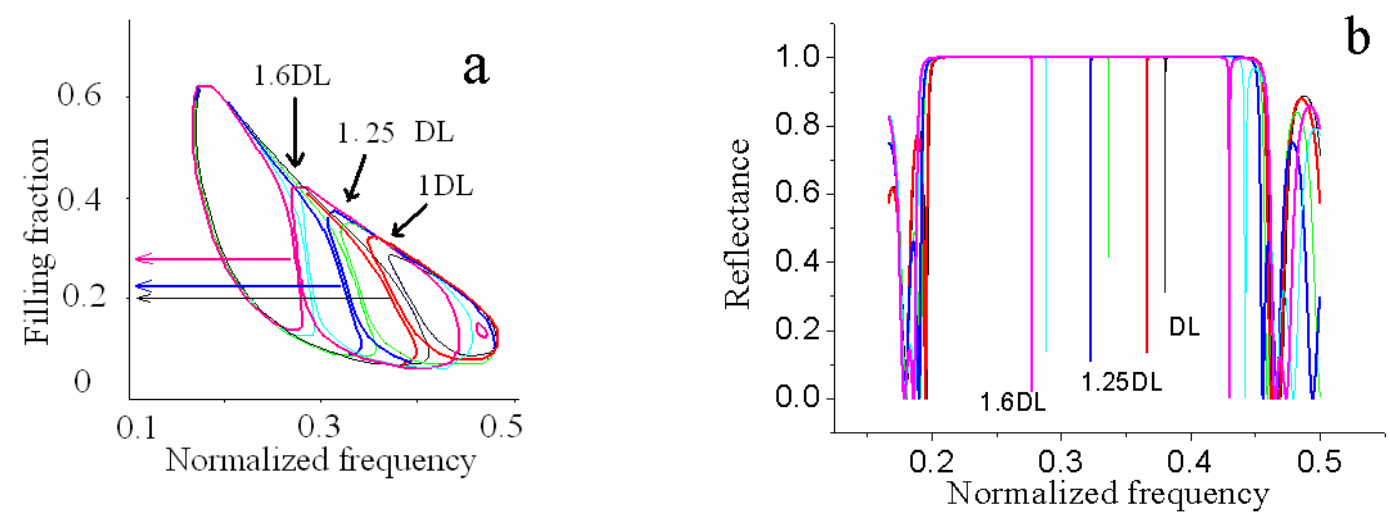

Fig. 2. 1D PC with tuning in the positions of the defect modes in the Ndef range from 1.5 (thin line) to 1.6 (thick line) corresponding to the width of the defect Ddef. The optical contrast in the BRs is 3.42/1 and the number of periods $m=$ 3: a) the diagram of the first (lower) PBG with defect mode areas; b) reflectance spectra showing the first (lower) PBGs, calculated for the various filling factors. The optimal filling factor for structures with $D d e f=\mathrm{DL}$ is equal to 0.2 , for $D d e f=1.25 \mathrm{DL}$ is 0.23 , for $D d e f=1.6 \mathrm{DL}$ is 0.3 . The beam falls in the normal plane.

Thus, the advantage of the GM approach is that the design of ODB from the point of view of technical realisation, including the minimisation of the number of periods $m$, is possible. In this work it is shown that a PC with medium contrast with ODB can be formed from photonic structures with the number of periods $m>20$ and $f$ varying in the range of 0.4 to 0.85 . The GM of PC with defect modes enables us to visualize the region of the defect modes and to optimize the parameters of both the PC and the defect.

\section{EXPERIMENT}

Three types of samples were analyzed in this study: i) conventional 1D PC, ii) 1D PC with micro-cavity and iii) interdigital 1D PC structure based on SOI. All grooved structures with cavity were fabricated from (110) oriented silicon with resistivity of 1-10 $\mathrm{Ohm} \mathrm{cm}$ by etching in hot $\mathrm{KOH}$ solution through a surface oxide mask designed according to the calculated geometrical parameters (for details of fabrication procedure see ${ }^{22}$ ). The depth of etching was varied from 20 $\mu \mathrm{m}$ to $40 \mu \mathrm{m}$ depending on the type of the structure. For the demonstration of the thermo-tuning effect for the PBG edge the conventional 1D PC with the lattice constant $A=2.8 \mu \mathrm{m}$, filling factor $D_{S i} / A=0.4$ and number of periods $m=2$ has been used (see Fig. 3 a). The micro-cavity structure with a central groove of width $D d e f=3.2 \mu \mathrm{m}$ and BRs on both sides with a lattice period $A=3 \mu \mathrm{m}$ (Si wall thickness $D_{S i}=1.6 \mu \mathrm{m}$ and groove thickness $D_{\text {air }}=1.4 \mu \mathrm{m}$ ) has been used for experimental demonstration of the effect of temperature on the cavity mode position. The cavity structure with the lowest number of periods was found to be the most suitable for the experiments on tuning. A schematic of the structure under investigation is shown in Fig. 3 b. 

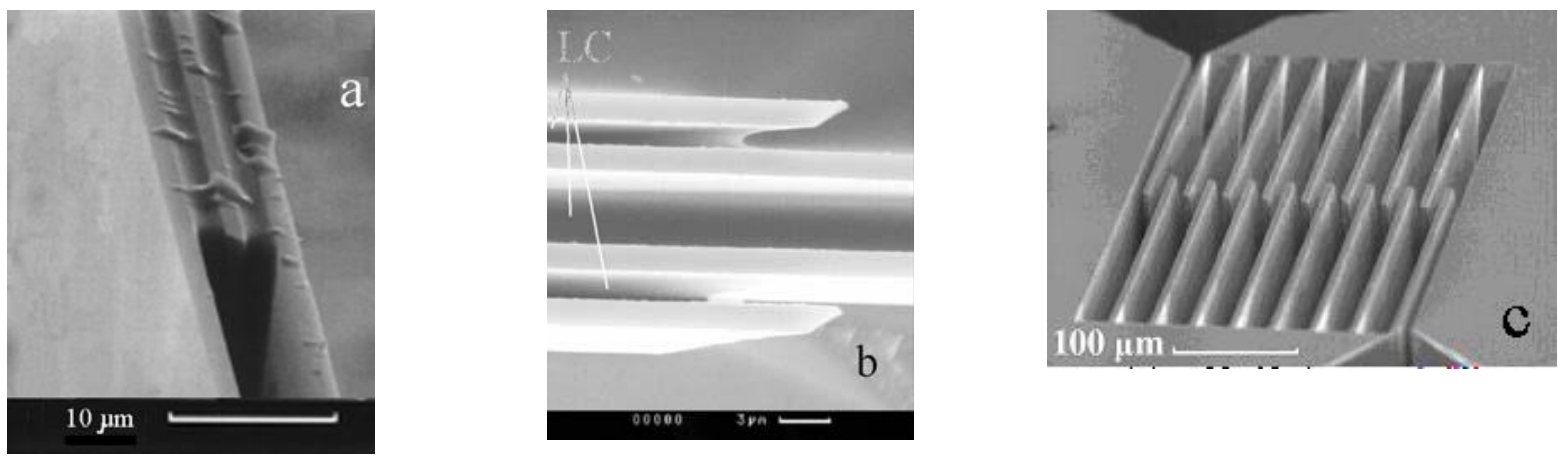

Fig.3 SEM images of 1D PC composite structures based on Si-LC a) conventional, b) micro-cavity, and c) inter-digital on SOI wafer.

The inter-digital grooved silicon structures for demonstration of electro-tuning effect have been formed on silicon-oninsulator (SOI) platform. SEM image of one of these structures is shown in Fig. 3 c. The initial SOI wafer from Si-Mat (Germany) was prepared from (110)-oriented silicon. It consists of a $40 \mu \mathrm{m}$ thick upper Si layer doped with phosphorus, $\mathrm{SiO}_{2}$ insulating layer of $2 \mu \mathrm{m}$ thickness and a silicon substrate. The stripes were arranged in an inter-digital configuration and after the etching these formed sixteen silicon ridge-electrodes in turn with grooves. The groove sidewalls, formed of (111) Si planes, are vertical and have an optically smooth surface. The region of electrode overlapping was used to apply an external voltage to the LC filler. The fabricated chips were mounted onto a foil-clad Gertinax plate, and the Si electrodes were connected with outer pads by means of thin metal wires attached to the chip contact areas by means of a silver paste. A frame around each chip provided a confined volume in common with the grooves and was designed to prevent LC leakage from the structure.

The commercial nematic LC mixture E7, based on cyano-biphenyl, with birefringence $\Delta n=0.2$ in the infrared spectral range was used for infiltration ${ }^{23}$. Infiltration of the grooves was performed at room temperature. Reflection spectra measurements have been performed in the range 1.5 - $15 \mu \mathrm{m}$ using an FTS 6000 FTIR spectrometer in conjunction with a UMA IR microscope. The aperture of the focused beam was $20 \times 20 \mu \mathrm{m}^{2}$, the spectral resolution was $8 \mathrm{~cm}^{-1}$ and the number of scans was 256. The home-made sample holder allowed positioning of the sample in $x-y$ and $z$ directions in order to attain normal incidence of light onto the $\mathrm{Si}$ wall of the structure. To vary the temperature a micro-heater (resistor) was attached to the sample. After the sample was placed in the correct position under the IR microscope, a micro-thermocouple was attached to the sample using a special holder. The reflection spectra were then measured for $E$ and $H$-polarisation at room temperature and at $\mathrm{T}=70^{\circ} \mathrm{C}$. The re-orientation of liquid crystal molecules due to the electrotuning and thermo-tuning effect were also confirmed by polarized optical microscopy and micro-Raman spectroscopy.

\section{RESULTS AND DISCUSSION}

\subsection{Thermo-tuning:}

Tuning of the $P B G$ edge

With regard to the thermo-tuning process it is important to obtain good initial alignment of the LC in the grooved $\mathrm{Si}$ matrix. A large number of LC alignment methods exist for normal LC cells but the application of these methods for PCLC composites is restricted by the microscopic dimensions of the surface where the alignment must be obtained. This is another challenging task, which we are going to explore in our future work. In this work the alignment of nematic LC in grooved Si structures was achieved simply by the capillary flow effect, which is not highly reproducible. Due to this fact, structures with a reasonably good planar alignment were selected, out of a large number of fabricated structures, for demonstration of the thermo-tuning effect. 
Figures $4 \mathrm{a}, 4 \mathrm{~b}$ and $4 \mathrm{c}$ show the reflection spectra vs. the wavenumber over the entire spectral range. The pronounced shift between $E$ - and $H$-spectra resulted from the anisotropy of the LC (Fig. 4a). The fact that the $E$-spectrum is shifted to a higher wavelength leads us to the conclusion that the director of the LC molecules is aligned along the grooves (planar homogeneous alignment). Moreover, both spectra show the characteristic LC vibrational bands at $v=2222 \mathrm{~cm}^{-1}$ $(4.5 \mu \mathrm{m}), 1600 \mathrm{~cm}^{-1}(6.2 \mu \mathrm{m})$ and $1497 \mathrm{~cm}^{-1}(6.7 \mu \mathrm{m})$. The intensity of all these bands is significantly larger for $E$ polarisation than for $H$-polarisation. Bearing in mind that the transition dipole moment for all these bands is oriented along the long molecular axis we can conclude that the director aligns along the Si grooves. This type of alignment was observed in our previous work due to flow-control alignment during the filling of the grooves with LC.
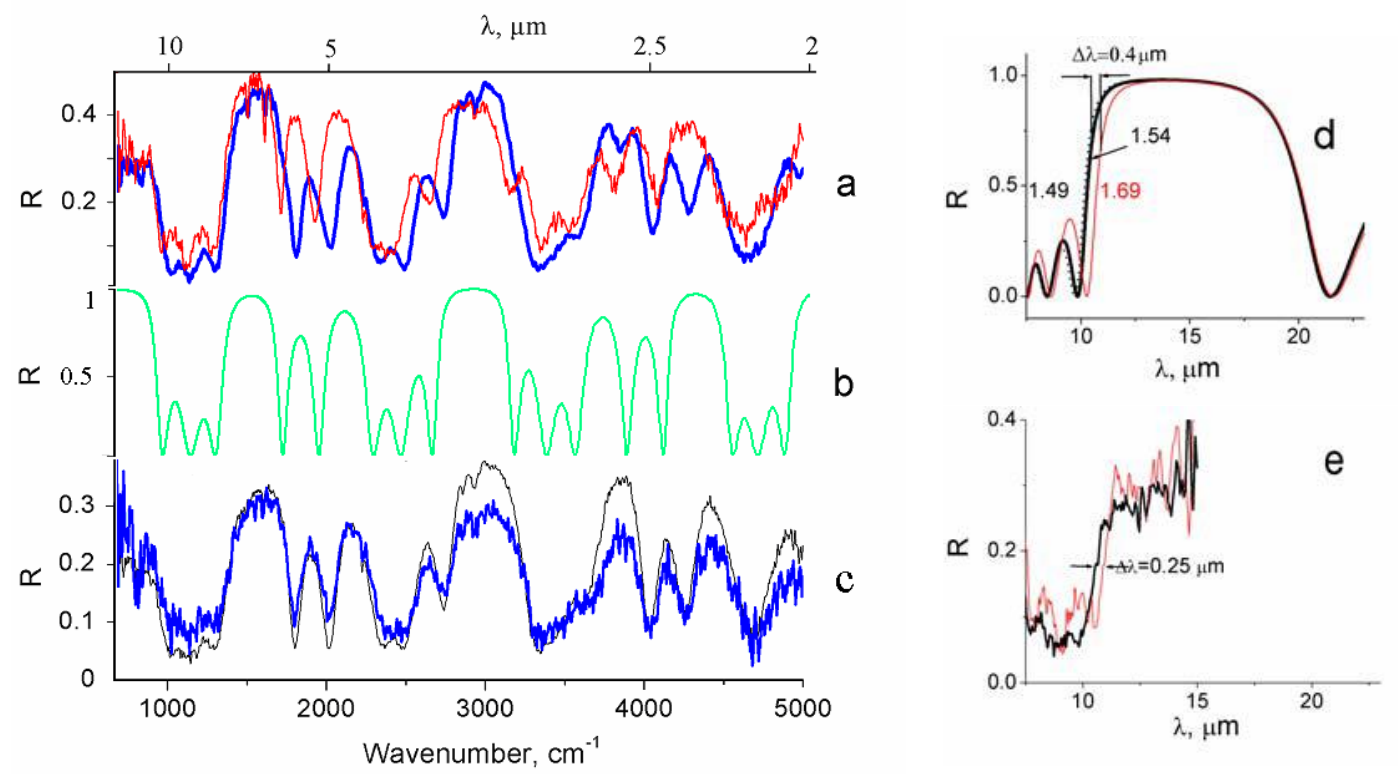

Fig. 4. Polarized reflection spectra ( $E$-thin line and $H$-thick line) of 1D PC-LC with lattice constant $A=2.8 \mu \mathrm{m}$, number of periods $m=2$ and filling factor $D_{S i} / A=0.43$ at a) room temperature and c) $\mathrm{T}=70^{\circ} \mathrm{C}$. b) Calculated spectrum for $E$-polarisation as the best fit to the spectrum shown in a), d) calculated reflection spectra for $E$-polarisation with a shift of the bandgap edge as a result of the thermo-tuning effect $(\Delta \lambda=0.4 \mu \mathrm{m}$ and $\Delta \lambda / \lambda=3.6 \%$ ), figures beside each line show the corresponding refractive index value of LC; e) experimental reflection spectra with shift of the bandgap edge of $\Delta \lambda=0.25 \mu \mathrm{m},(\Delta \lambda / \lambda=2.3 \%)^{13}$.

By fitting the experimental reflection spectra, shown in Fig. 4a and 4c, we obtained the refractive indices of LC for different temperatures. During the fitting we used the parameter $D_{S i}=1.26 \mu \mathrm{m}$, obtained by simulation of the spectrum of an empty structure. As criteria for fitting we used the simultaneous coincidence at the edges, maxima and minima for the main and the nearest secondary reflection bands, as well as for other local bands (more than 10 bands in total). The resultant spectrum for the best fitting for $E$-polarisation is shown in Fig. $4 \mathrm{~b}$ for comparison. As a result of this fitting the following value of refractive index at room temperature has been obtained: $n_{L C}=1.70 \pm 0.02$ for $E$-spectra and $n_{L C}=1.53 \pm 0.02$ for $H$-spectra. These results agree very well with published data for ordinary, $n_{o}$ and extraordinary, $n_{e}$ refractive indices $\left(n_{o}=1.49\right.$ and $\left.n_{e}=1.69\right)$ of LC E7 in the mid-infrared region ${ }^{23}$.

The reflection spectra for $E$ - and $H$-polarisation measured at $\mathrm{T}=70{ }^{\circ} \mathrm{C}$ are presented in Fig. $4 \mathrm{c}$. We can see that in this case both spectra are coincident, which confirmed the absence of anisotropy in the LC alignment. The refractive index $n=1.53 \pm 0.03$ obtained after the fitting of these spectra is in good agreement with $n$ for the isotropic phase of E7 $\left(n_{i}=1.54\right)$. A comparison of $E$ spectra for $\mathrm{T}=23{ }^{\circ} \mathrm{C}$ and $\mathrm{T}=70{ }^{\circ} \mathrm{C}$ is given in Fig. $4 e$ and this demonstrates the experimental evidence of a change in reflection spectra due to change in the refractive index. As one can see, the $E$ spectrum is shifted to the short wavelength range as a result of heating the LC to the isotropic phase. The experimental 
value for the shift, $\Delta \lambda=0.25 \mu \mathrm{m}\left(\Delta \lambda / \lambda_{\text {edge }}=2.3 \%\right)$ was obtained for the main band gap (at $\left.\lambda_{\text {edge }}=11 \mu \mathrm{m}\right)$. We believe that the deviation of the $\Delta \lambda$ value from the calculated one $(0.40 \mu \mathrm{m})$, shown in Fig. $4 \mathrm{~d}$, is due to some deformation of the interference band edge, though the results of fitting of the refractive index are in good agreement with known values for $n_{e}$ and $n_{o}$.

\section{Tuning of the defect mode}

The reflection spectrum for the structure shown in Figs. 3 b and 5 with empty grooves was measured first (see Fig. 6 b) and this was followed by the fitting procedure of the obtained spectrum using the TMM method. This procedure enabled us to obtain the thickness values of the Si walls $\left(D_{S i}=1.57 \mu \mathrm{m}\right)$, the grooves $\left(D_{g r}=1.43 \mu \mathrm{m}\right)$ and the defect $(D d e f=3.1 \mu \mathrm{m})$. As one can see, the geometrical parameters found by fitting of the experimental curve are very close to those of the designed structure. The best fit to the experimental spectrum is presented in Fig. $6 b$ by the dotted line.

Following this, all grooves were infiltrated with the LC and the reflection spectra of the composite structure were measured at different polarisations at $\mathrm{T}=20^{\circ} \mathrm{C}$. Fig. $6 c$ shows normalised reflection spectra of the second-order stop-band in two orthogonal polarisations. It demonstrates a slight shift of the band edges and more pronounced shift of the resonance peak between the spectra for $E$ and $H$-polarization. This anisotropy confirms the existence of a certain alignment of the liquid crystal molecules inside the grooves. The results of fitting show that $n_{L C}$ for $E$-polarisation is 1.64 , whereas for the $H$-polarisation it is 1.52 . Both values differ from the values for the extraordinary, $n_{e}=1.69$ and ordinary refractive, $n_{o}=1.49$ indices of nematic LC E7. This result shows that the $E$-spectrum is formed due to the higher refractive index and consequently, due to the predominantly homogeneous planar alignment of the LC director with respect to the Si sidewalls. Similar to the results obtained in the previous paragraph both spectra show characteristic LC vibrational bands at $\mathrm{v}=2222 \mathrm{~cm}^{-1}, 1600 \mathrm{~cm}^{-1}$ and $1497 \mathrm{~cm}^{-1}$. The intensity of all these bands is larger for $E$-polarization than for $H$-polarization. Therefore, we can confirm that the director aligns along the Si groove (Fig. $5 \mathrm{a}$ ). The deviation of the observed LC refractive indices from data published in the literature for $n_{e}$ and $n_{o}$ is due to the non-perfect orientation (quasi-planar) of the LC director along the groove length.
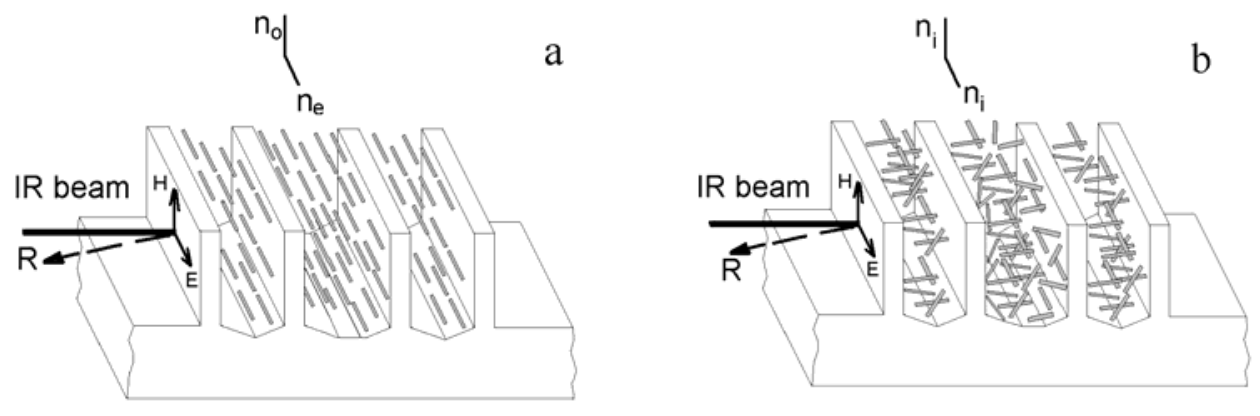

Fig.5. Schematic of in-plane polarized FTIR reflection measurements of 1D PC with defect and the direction of the incident and the reflected light beam. The orientation of LC molecules and the vectors $n$ of LC are shown for the case of a) planar and b) isotropic alignment.

At $\mathrm{T}=70{ }^{0} \mathrm{C}$ the reflection spectra measured for $E$ - and $H$-polarization are coincident (Fig. $6 \mathrm{~d}$ ). In this case both spectra confirmed the absence of any anisotropy in the LC (Fig. 5 b). The refractive index $n_{L C}=1.53$ obtained from fitting of these spectra is in good agreement with the value for the isotropic phase of E7 $\left(n_{L C}=1.54\right)$. A comparison of the $E$-spectra for $\mathrm{T}=20{ }^{\circ} \mathrm{C}$ and $\mathrm{T}=70{ }^{\circ} \mathrm{C}$ demonstrates the experimental evidence of change in peak position due to variation in refractive index. Note that the peak position for $H$-polarization at $20^{\circ} \mathrm{C}$ is practically the same as at $70{ }^{\circ} \mathrm{C}$. That is because of the minor difference between their refractive indices (1.53 and 1.52, respectively). As one can see, the $E$ spectrum is blue-shifted as a result of the LC transition to isotropic phase under heating and decreasing of the refractive 
index. An experimental value for the peak shift, $\Delta \lambda=0.17 \mu \mathrm{m}(\Delta \lambda / \lambda=2.0 \%)$ was obtained. We believe that the deviation of the $\Delta \lambda$ value from that calculated, $0.26 \mu \mathrm{m}$, shown in Fig. $6 a$, is due to quasi-planar initial LC alignment.

Thus, in this section we have shown that for a cavity in the grooved Si-LC 1D composite photonic structure the shift of the peak position under heating is rather large. This occurs due to transition of the LC from the planar to the isotropic state of alignment.
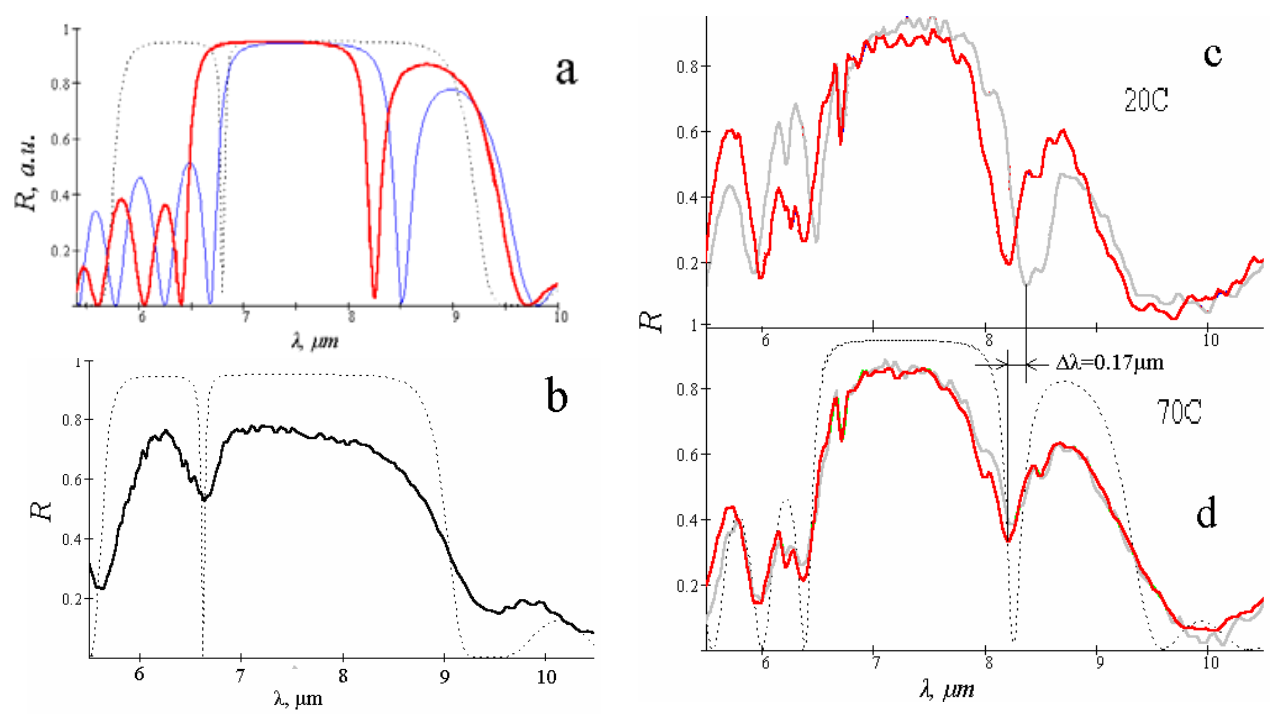

Fig.6. a) Calculated reflection spectra in E-polarisation of second-order stop-band for 1D PC with empty grooves (dotted line) and with grooves filled by LC at $n_{L C}=1.54$ (thick line) and $n_{L C}=1.69$ (thin line) for isotropic and planar alignment, accordingly. The Si filling fraction is $f=0.53$, normal incidence of light. b) Calculated (dotted line) and experimental (solid line) reflection spectra of second-order stop-band of empty 1D PC with defect. Reflection spectra of PC with $\mathrm{LC}$ at c) $20^{\circ} \mathrm{C}$ for $\mathrm{E}$ (black line)- and $\mathrm{H}$ (gray line)- polarization, and d) at $70{ }^{\circ} \mathrm{C}$, experimental shift of the defect peak $\Delta \lambda=0.17 \mu \mathrm{m}$ (relative shift $\Delta \lambda / \lambda=2 \%$ ).

\subsection{Electro-tuning:}

\section{Polarized optical microscopy and micro-Raman measurements}

The electro-optical effect was studied by means of polarized microscopy and micro-Raman techniques under $d c, a c$ or pulsed voltage with signal frequency in the range 20-100 Hz. Firstly, the Freedericksz transition (reorientation of LC molecules from planar to homeotropic alignment) was registered with microscope in the reflection mode under crossed polarizers. Images and movies were recorded by CCD web-camera adjusted to the microscope.

Raman measurements were carried out in backscattering geometry using Renishaw micro-Raman system 1000 with Peltier cooled CCD camera. The polarised $514.5 \mathrm{~nm}$ line of an $\mathrm{Ar}^{+}$laser with power of $5 \mathrm{~mW}$ focused to a spot size of about $1 \mu \mathrm{m}$ has been used for the excitation. In order to obtain the intensity of the band at $1606 \mathrm{~cm}^{-1}$ the experimental spectra, collected at different polarizations, were fitted by Lorentzian function. Schematic of the experiment is shown in Figs.7 a and 7 b. The polarization $\boldsymbol{E}$ of the incident laser light was always identical (in the horizontal direction in Fig. 7), while the sample was rotated by $90^{\circ}$. So the grooves could be oriented either parallel or perpendicular to the light polarization vector $\boldsymbol{E}$, i.e., in $H$ or $V$ position in the picture. The incident light was focused onto the central part of the groove infiltrated with LC between the electrodes. After rotating the crystal from the position $H$ to the position $V$, the exposed spot was fixed at the same point. As a rule, in polarized Raman spectroscopy, the coordinate system is rigidly attached to the sample. The scattered light intensity $I_{i j}$ has two subscripts defining the polarization of scattered $(i)$ and incident $(j)$ light. The peak intensities $I_{x x}, I_{y y}, I_{x y}$ and $I_{y x}$ were registered at different polarizations of the incident and 
scattered light at different values of electric field directed along the $\mathrm{Y}$ axis. In such notation, the depolarization ratios for the two sample orientations can be written as $R_{H}=I_{y x} / I_{x x}$ and $R_{V}=I_{x y} / I_{y y}$.
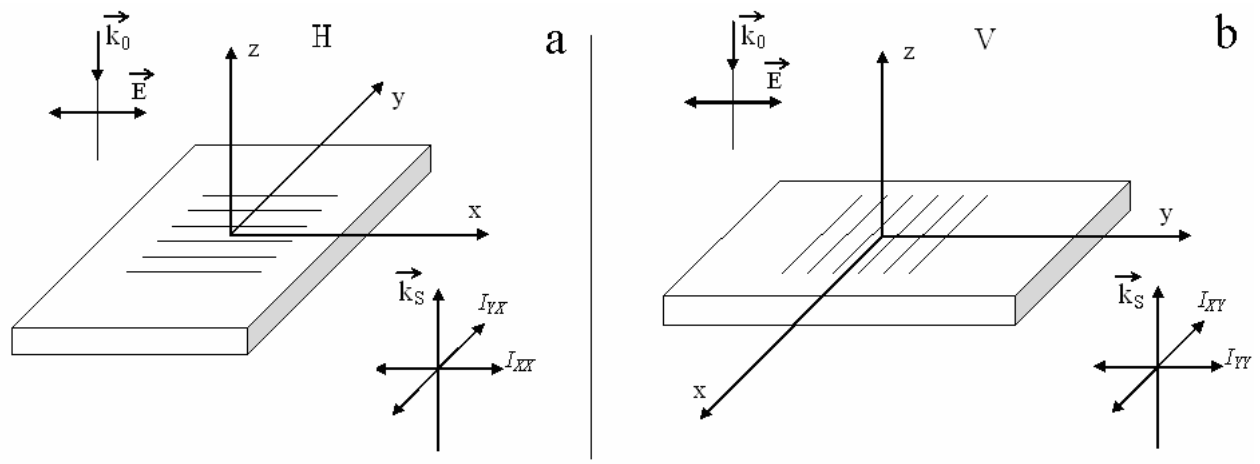

Fig.7.Schematic diagram of the Raman scattering measurements: a) position of the grooves $H$, parallel to the polarization vector $\boldsymbol{E}$ of incident light and b) position of the grooves $V$, perpendicular to the polarization vector $\boldsymbol{E}$ of incident light; $\mathbf{k}_{0}$ and $\mathbf{k} s$ are the wave vectors of incident and scattered light, respectively.

Silicon ribs were used to apply an external voltage to the LC filler (see Fig. 8 a). The electro-optical effect was studied under a DC or saw-shaped voltage of frequency $50 \mathrm{~Hz}$. Freedericksz transition was registered with polarized optical microscopy in the reflection mode when the polarizer and analyzer were crossed. At $\mathrm{V}=0$ the $\mathrm{LC}$ was bright in the region of the overlapping electrodes (Fig. 8 b), it became dark (Fig. 8 c) under a voltage higher than the threshold voltage of $1.41 \mathrm{~V}$ for LC E7 ${ }^{24}$, and turned bright again when the voltage was turned off. The characteristic switching time measured for the electro-tuning effect was $\sim 30 \mathrm{msec}$.

The most intense band observed in Raman spectrum at $1606 \mathrm{~cm}^{-1}$ (see Figs. 9a and 9b) is assigned to C-C aromatic stretching vibration oriented along the molecular axis. This particular band is usually used for the determination of the order parameter in LCs based on cyanobiphenyls ${ }^{22-25}$. The intensity of $I_{x x}$ component is larger than the intensity of $I_{y y}$ component for the Raman spectra in Fig. 9 a. This demonstrates an initial planar alignment of the LC molecules along the grooves ( $x$-axis), which is in agreement with the results obtained in our previous work ${ }^{22}$ for similar structures. When an external voltage is applied the initial planar alignment is changed to the pronounced homeotropic alignment along the Y-axis with $I_{y y}>I_{x x}$. Fig. $9 \mathrm{~b}$ shows the changes in the intensity of the polarised components of scattered light for the Raman peak at $1606 \mathrm{~cm}^{-1}$. The voltage dependence of the dichroic ratio $N=I_{y y} / I_{x x}$ is shown in Fig. $9 \mathrm{c}$. It is seen that the threshold voltage for Freedericksz transition is reached between $2 \mathrm{~V}$ and $3 \mathrm{~V}$ and is in agreement with the known value for nematic LC E7 ${ }^{24}$.
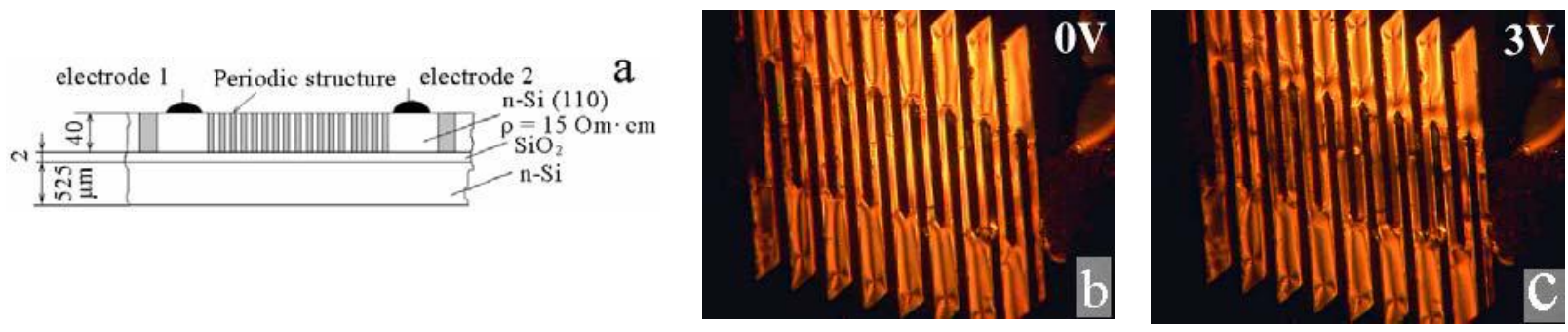

Fig. 8. a) Schematic of grooved silicon structure on SOI wafer. Electro-optical effect shown for the structure of period 16 $\mu \mathrm{m}$ (optical microscope image under crossed polarizers). Black stripes are silicon electrodes, light regions are grooves filled with LC. Snapshots were obtained from the movies recorded under applied $d c$ voltage of b) $0 \mathrm{~V}$ and c) $3 \mathrm{~V}$. 

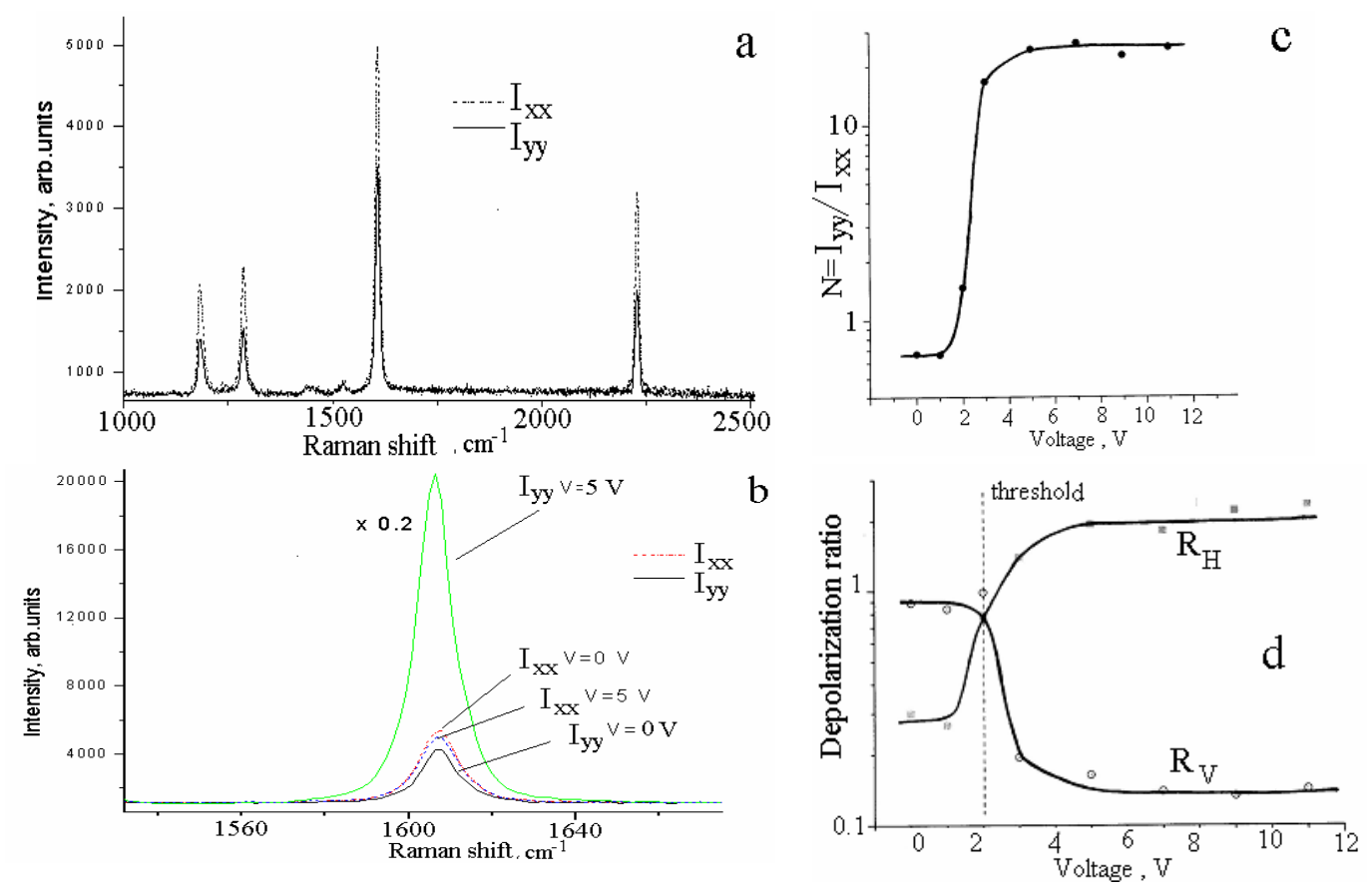

Fig.9. Raman spectra for sample $a 8 d$ : a) the components $I_{x x}$ and $I_{y y}$ at $\mathrm{V}=0$, b) influence of electric field on the amplitude of scattered peak at $1606 \mathrm{~cm}^{-1}$ Voltage dependence of (a)- anisotropic ratio of Raman components $N=I_{y y} / I_{x x}$ and (b)depolarisation ratios $R_{V}$ and $R_{H}{ }^{25}$.

The depolarization and anisotropic ratios calculated from the measured intensities of scattered light are shown in Fig. 9d versus applied voltage. It is known that for the case when the director is oriented along the $\mathrm{X}$ axis, the anisotropic ratio $N=I_{y y} / I_{x x}<1$ at $R_{V}>R_{H}$, and for a well ordered LC $R_{V}>1^{25,26}$. As is seen from the $N, R_{V}$ and $R_{H}$ values at $V=0$ the initial LC alignment for sample under investigation is not pronounced and reproducible. It indicates just a feebly marked planar alignment along the grooves. This manifests weak anchoring forces between the LC molecules and Si walls. Rectangular pulses of the voltage force LC molecules to align along the field in Y-direction. It can be seen from Fig. $9 \mathrm{~b}$ that the amplitude $I_{y y}$ increases by factor of 30 when rectangular pulses with an amplitude of $V=5 \mathrm{~V}$ are applied to the electrodes, whereas $I_{x x}$ component decreases only by factor of 1.11 . The ratio $R_{V}$ decreases, whereas $R_{H}$ increases as the electrical field strengthens, which indicates rise in the fraction of molecules oriented along the electric field (perpendicular to the grooves). The saturation of the curves at $V>4 \mathrm{~V}$ suggests that the formed alignment of LC molecules in the grooves does not change any more. It is evident from the drastically changed values for the depolarization ratios $R_{H}>2, R_{V}<0.17$ and from high values of $N>16$ that the orientation becomes pronounced homeotropic with respect to $\mathrm{Si}$ walls. The electro-optical effect was evaluated as a ratio of $N\left(V_{s}\right)$ under the applied voltage in the saturation region to the initial value $N(V=0)$ and found to be around 18 for the studied sample.

Therefore, the periodic silicon structures on SOI platform with inter-digital electrodes obtained by deep anisotropic etching are suitable matrices for formation of composite electro-tunable devices. The structures infiltrated with LC provide possibility to control refractive index of the filler by external voltage and therefore to change the optical characteristics of the structures.

\section{FTIR measurements}

To demonstrate the electro-tuning effect on the PBG position, we have chosen a periodic structure with a minimal number of periods $m=2$ (Fig.10 a). As will be shown below, this number of periods is enough to reveal the electro-optical effect on the shift of the stop bands. For experimental convenience we have performed calculations of the reflection spectra, $R$, for structures with a lattice period $A=D_{S i}+D_{L C}=6 \mu \mathrm{m}$ (at $D_{S i}=2.4 \mu \mathrm{m}$ and $\left.D_{L C}=3.6\right)$. Spectra $R$ were calculated using the TMM at normal incidence of light for the model shown in Fig. 10 a at $n_{S i}=3.42, n_{L C}=1.56, m=20$ (Fig. $10 \mathrm{~b}$, 
dotted line) and $m=2$ (Fig. $10 \mathrm{~b}$, solid line) in order to demonstrate the difference in the reflection spectra of a two-period structure compared to a multi-period 1D PC. In both cases the first PBG is located outside of the working region of our MCT detector, which has a range of 1.5 to $15 \mu \mathrm{m}$, whilst the secondary PBGs are within this range and also have $R \approx 1$ due to the medium optical contrast, $n_{S i} / n_{L C}=3.42 / 1.56$. As can be seen from Fig. $10 \mathrm{~b}$ the secondary high reflection bands correspond to the position of PBGs for the multi-period 1D PC, but show slightly smaller values of $R$ maximum $(0.92-0.99)$ and less sharp edges.

Let us now model the variation of $n_{L C}$ in the grooves from the pseudo-isotropic $(n=1.56)$ to the homeotropic $(n=1.49)$ LC state and calculate the IR reflection spectra for the structure considered above, i.e. with $f=0.4$ and $m=2$. The calculated spectra $R$ are shown in Fig. $10 \mathrm{~d}$ in the wavelength range $4-16 \mu \mathrm{m}$. The presence of only one interference peak between the regions of high reflection at 9.5 and $13 \mu \mathrm{m}$ enables us to see the shift of the edges of these bands more clearly than for the structure with twenty periods shown in Fig. $10 \mathrm{~b}$. The interesting fact is that the shift of both the edges for each of these stop bands has been revealed in Fig. $10 \mathrm{~d}$. In order to understand this phenomenon we plotted PBG gap maps. These GMs are based on the calculation of the reflection spectra for all possible $f$ values (from 0.01 to 0.99 ) followed by the selection of regions, $\lambda$, with $R$ values satisfying the condition $R>z$, where $z$ is the criteria of the region selection. For the multiperiod PCs $z$ value is very close to 1 (for example, 0.99), for structures with two periods we chose $z=0.9$ in order to clearly demonstrate the shift of the PBG due to the influence of the electric field on LC orientation. A fragment of the GM constructed for the periodic structure with $m=2$ is presented in Fig. $10 \mathrm{c}$. As was expected, all regions of high reflection are blue shifted when the values of $n_{L C}$ are varied from 1.56 to 1.49 . By drawing lines at $f=0.4$ and $f=0.1$, which intersect a number of PBG regions, one can see three possible cases of PBG edge shift: i) only the short wavelength edge is shifted, ii) only the long wavelength edge is shifted and iii) both edges are shifted. Therefore, based on the gap map analysis, one can expect the appearance of different types of shifts of the reflection spectra edges. The value of $f=0.4$ was chosen taking into account the real technological conditions for the experimental verification of the electro-optical effect on one of the secondary bands of high reflection.

A silicon-on-insulator (SOI) wafer was prepared from (110)-oriented silicon (see schematic in Fig. $10 \mathrm{a}$ ). It consisted of a $40 \mu \mathrm{m}$ thick upper n-type Si layer $\left(\rho=15\right.$ Om.cm), a $2 \mu \mathrm{m}$ thick $\mathrm{SiO}_{2}$ insulating layer and a $500 \mu \mathrm{m}$ thick (110) silicon substrate. Structures with three vertical walls were etched in the top Si layer in a hot KOH solution through a surface oxide mask (see Ref. ${ }^{22}$ for details). The LC used was the nematic liquid crystal mixture E7. Infiltration into the grooves was performed at room temperature.

In order to apply an external voltage to the LC filler, the Si ribs were arranged in an inter-digital configuration. Si electrodes were connected to the outer pads by adhesing thin metal wires to the chip contact areas with a silver paste. The frequency and duration of the impulse were $100 \mathrm{~Hz}$ and $1 \mathrm{~ms}$, respectively. The reflection spectra measurements were performed using FTIR microscopy as described above. For FTIR measurements the applied electric field was $10 \mathrm{~V}$ for reliability. The response time estimated under applied square shaped $a c$ pulses of various frequencies was found to be around $30 \mathrm{~ms}$ which is in agreement with the known value for nematic LC E7.

Reflection spectra of the empty grooved Si matrix were measured at $\mathrm{H}$ and E polarized light, this was followed by the fitting procedure using the TMM method. This procedure enables us to obtain more precise values of the wall and groove thicknesses. The grooved matrix was then infiltrated with LC E7 and the reflection spectra of the composite structure were measured at different polarisations and different voltages. The reflection spectra demonstrating the electro-optical effect for the investigated structure are shown in Fig. 10 e (for simplicity only the E-polarisation is shown). However exactly the same effect has been observed for H-polarized spectra.

The comparison of the experimental and calculated spectra demonstrates a good agreement between the positions of the main reflection bands. It is important to note that the shift of the experimental reflection bands at $0 \mathrm{~V}$ and $10 \mathrm{~V}$ was observed in the whole spectral range for practically all bands of high reflection as was predicted from the calculated spectra shown in Fig. 10 d. The electro-optical effect was estimated for the long wavelength edge of the calculated (shown in detail in Fig. $10 \mathrm{f}$ ) and experimental (Fig. $10 \mathrm{e}$ ) secondary stop-band in the region of $\sim 10 \mu \mathrm{m}$. The calculated shift is $0.22 \mu \mathrm{m}$ and $\Delta \lambda / \lambda=0.22 / 10.15=2.2 \%$ in relative shift units. The experimental electro-optical shift estimated in the same way is $0.16 \mu \mathrm{m}$ and $1.6 \%$ respectively. 
Thus, in this section we have shown that for grooved Si-LC 1D composite photonic structures the shift of the stop-band edge under an applied electric field is quite large even for a transition of the LC from quasi-isotropic to homeotropic alignment. The relative shift of the band edge at half intensity in the region of $10 \mu \mathrm{m}$ was estimated as being $2.2 \%$ theoretically and $1.6 \%$ experimentally.
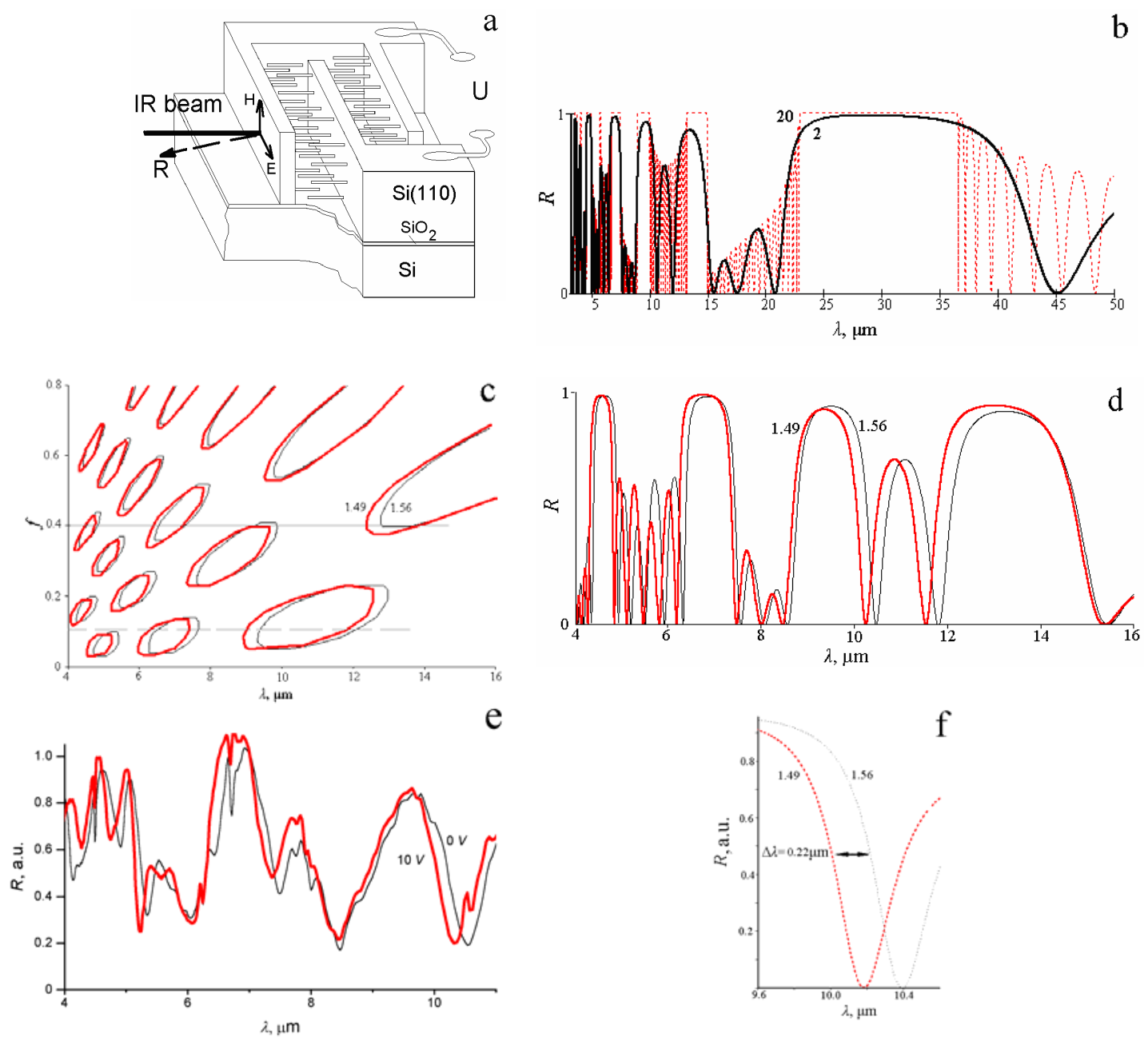

Fig. 10. a) Schematic of a composite photonic structure with two lattice periods based on a SOI platform with homeotropic alignment of LC.b. The calculated reflection spectra of 1D PCs based on grooved silicon with $f=0.4$ and number of periods $m=2$ (solid line) and $m=20$ (dotted line) filled with liquid crystal $\left(n_{L C}=1.56\right)$. c) The calculated reflection spectra for a composite photonic structure with $m=2$ with refractive index values $n_{L C}=1.56$ (thin line) and $n_{L C}=1.49$ (thick line) LC. d) The gap map fragments of PBGs with high reflection regions $(R>0.90)$ calculated for a photonic Si-LC structure with $m=2, A=6 \mu \mathrm{m}, n_{L C}=1.49$ and 1.56 at normal incidence of light. e) Experimental and $\mathrm{f}$ ) calculated (part of the secondary stop-band) the E-polarised reflection spectra, without $(0 \mathrm{~V}$, thin line) and with (10V, thick line) an applied electric field for composite photonic structure based on grooved Si filled with LC E7 with two lattice periods ${ }^{16}$.

\section{CONCLUSION}

In this paper, the results obtained on design, fabrication and characterisation of tunable one-dimensional photonic structures based on Si and LC are summarised. The gap map approach has been elaborated for the analysis of the defect mode and omnidirectional reflection and the comparison with the results obtained from the band diagram method was 
performed. It was shown that photonic band gap and defect mode tuning can be achieved by variation of: i) the structure of the material (optical contrast and the number of periods), ii) the angle of incidence and beam polarisation and iii) the refractive index of the defect (introduction of a liquid crystal and its re-orientation). The optical properties of fabricated grooved Si structures infiltrated with liquid crystal E7 were simulated using a transfer matrix method and have been verified experimentally using FTIR microscopy. The calculations predict a relative shift, $\Delta \lambda / \lambda_{\text {edge, }}$ of the PBG edge to be equal to $3.6 \%$ in the case of thermo-tuning, while the experiment demonstrated $\Delta \lambda / \lambda_{\text {edge }}=2.3 \%$ for the main PBG edge near $\lambda=11 \mu \mathrm{m}$. It was shown that for a cavity in the grooved Si-LC 1D composite photonic structure, infiltrated with LC E7, the relative shift of the peak position under heating is about $2 \%$. Both of the above mentioned effects occur due to the transition of the LC from the planar to the isotropic state of alignment in the course of the heating. Periodic grooved silicon structures on SOI platform with inter-digital electrodes were used for the formation of composite electrotunable devices. The electro-tuning effect was obtained for composite photonic structures for the edge of the third photonic bandgap near $\lambda=10 \mu \mathrm{m}$ to be equal to $\Delta \lambda / \lambda=1.9 \%$. The characteristic switching time measured for the electrotuning effect is $\sim 30 \mathrm{msec}$, while the threshold voltage is $2 \mathrm{~V}$. The re-orientation of liquid crystal molecules due to the electro-tuning and thermo-tuning effect were also confirmed by polarized optical microscopy and micro-Raman spectroscopy.

\section{Acknowledgments}

This work has been supported by the Science Foundation Ireland Basic Research Grant Scheme (Grant 04/BR/P0698) and Programme of Russian Academy of Sciences "Optics and Laser Physics". The authors wish to express their appreciation to S. O'Neill and J. Ruttle for calculations.

\section{REFERENCES}

1 A.Liu, M. Paniccia, “Advances in silicon photonic devices for silicon-based optoelectronic applications," Physica E 35, 223-228 (2006).

2 R.Soref, "The Past, Present, and Future of Silicon Photonics," IEEE J.Selected Topics in Quant. Electr. 12(6) 16781687 (2006).

3 E. Yablonovitch, "Inhibited Spontaneous Emission in Solid-State Physics and Electronics," Phys. Rev. Lett. 58(20), 2059-2062 (1987).

4 S. John, "Strong localization of photons in certain disrdered dielectric superlattices," Phys.Rev.Lett., 58(23), 24862489 (1987).

5 J.D.Joannopoulos, R.D.Meade, R.D.Winn, Photonic Crystals. Princeton University Press, 1995.

6 K. Busch, S. Lölkes, R. Wehrspohn, H. Föll (eds.), Photonic Crystals. Advances in Design, Fabrication, and Characterization, Weinheim: Wiley-VCH, 2004.

7 Y. Fink, J. N. Winn, F. Shanhui, C. Chiping, J. Michel, J. D. Joannopoulos, and E. L. Thomas,"A dielectric omnidirectional reflector," Science 282, 1679-1682 (1998).

8 D.N. Chigrin, A.V. Lavrinenko, D.A. Yarotsky, S.V. Gaponenko, "All-Dielectric One-Dimensional Periodic Structures for Total Omnidirectional Reflection and Partial Spontaneous Emission Control," J. Lightwave Technology, 17( 11) 2018-2024 (1999).

9 K. Busch and S. John, Phys. Rev. Lett. "Liquid-Crystal Photonic-Band-Gap Materials: The Tunable Electromagnetic Vacuum," 83(5), 967-970 (1999).

10 S. Leonard, J. Mondia, H. van Driel, O. Toader, S, John, K. Busch, A. Birner, U. Gosele, and V. Lehmann, "Tunable two-dimensional photonic crystals using liquid-crystal infiltration,” Phys. Rev. B 61(4) R2389 -2392 (2000).

11 Ch. Schuller, F. Klopf, J. P. Reitmaier, M. Kamp, and A. Forchel, "Tunable photonic crystals fabricated in III-V semiconductor slab waveguides using infiltrated liquid crystals," Appl. Phys. Lett., 82(17), 2767-2769 (2003).

12 G. Mertens, T. Roder, R. Schweins, K. Huber, and H-S. Kitzerowa," Shift of the photonic band gap in two photonic crystal-liquid crystal composites," Appl. Phys.Lett. 80(11), 1885-1887 (2002).

${ }_{13}$ T.S. Perova, V.A. Tolmachev, E.V. Astrova, Ju. Zharova and S.M. O’Neill, "Tunable one-dimensional photonic crystal structures based on grooved Si infiltrated with liquid crystal E7," Phys. Stat. Sol. (c) 4 (6) 1961-1965 (2007).

14 M. Haurylaua, S. P. Anderson, K. L. Marshall, P. M. Fauchet, "Electrical modulation of silicon-based twodimensional photonic bandgap structures," Appl. Phys. Lett. 88, 061103 (2006). 
15 E. Astrova, T. Perova, Ju. Zharova ,S.Grudinkin, V. Tolmachev, V.Melnikov, “Electro-tunable one-dimensional photonic crystal structures based on grooved silicon infiltrated with liquid crystal," J. Luminescence 121 (2), $298-300$ (2006).

16 V.A. Tolmachev, E.V. Astrova, T.S. Perova, J.A. Zharova, S.A. Grudinkin, V.A. Melnikov, "Electro-tunable inplane one-dimensional photonic structure based on silicon and liquid crystal”, App. Phys. Lett., 90, 011908 (2007).

17 P. Yeh, Optical Waves in Layered Media. New York: Wiley, 1988.

18 M. Born, E. Wolf, Principles of Optics", Pergamon Press, Oxford-London, 1964.

19 V. A. Tolmachev, T. S. Perova, and R. A. Moore, "Method of construction of composite one-dimensional photonic crystal with extended photonic band gaps," Opt. Express, 13(21), 8433-8441 (2005).

20 V. A. Tolmachev, T. S. Perova, and K. Berwick, "Design criteria and optical characteristics of one-dimensional photonic crystals based on periodically grooved silicon,” Appl. Opt., 42, 5679-5683 (2003).

21 V.A. Tolmachev, T.S. Perova, and K. Berwick. "Design of $1 \mathrm{D}$ composite photonic crystals with an extended photonic band gap,” J. Appl. Phys. 99, 033507 (2006).

${ }_{22}$ V. A. Tolmachev, E. V. Astrova, Yu. A. Pilyugina, T. S. Perova, R. A. Moore, and J. K. Vij, "1D photonic crystal fabricated by wet etching of silicon", Optical Materials, 28(5) 831-835 (2005).

23 L. C. Khoo, "The Infrared Optical Nonlinearities of Nematic Liquid Crystals and Novel Two-wave Mixing Processes", J. Mod. Opt. 37, 1801 (1990).

24 S.-T. Wu, "Birefringes dispersion of liquid crystals", Phys. Rev. 33(2) 1270-1274 (1986).

25 E.V. Astrova, T.S. Perova, S.A. Grudinkin, V.A. Tolmachev, Yu.A. Pilyugina, V.B. Voronkov, "Polarised Infrared and Raman Spectroscopy Studies of the Liquid Crystal E7 Alignment in Composites based on Grooved Silicon", Semiconductors, 39(N7), 759-767 (2005).

26 S. Jen, N. A. Clark, P. S. Pershan, and E. B. Priestley, "Polarized Raman scattering studies of orientational order in uniaxial liquid crystalline phases", J. Chem. Phys., 66, 4635-4661 (1977). 